\title{
The Study on Decomposition of Fluctuant Wind Power for Wind Power Suppression
}

\author{
Peng Yu, Shumin Sun, Yan Cheng, Guanglei Li, Yong Zhang \\ State Grid Shandong Electric Power Research Institute, \\ Jinan, 250002, China
}

\begin{abstract}
Based on the turbulence model, the volatility of realtime wind speed is discussed, which is composed of the average component and fluctuant component. By deriving the probability density function of fluctuant wind speed and investigating the probability density curve, we decompose the fluctuant wind power into the steady fluctuation and peak fluctuation. The steady fluctuation has the low power value and leads to great energy variation. However, the peak fluctuation whose power value is high induces the small energy fluctuation. At the end of this paper, we give a calculation example to illuminate the decomposition of wind power fluctuation. Based on the wind power decomposition, the proper suppression method and control algorithm can be developed to not only realize the real-time wind power suppression but also reduce the investment cost.
\end{abstract}

Keywords-power decomposition; turbulence model; wind speed; fluctuant wind power

\section{INTRODUCTION}

With the development in wind turbine technologies, the wind power generation has become competitive with other conventional power generation patterns. In order to enhance the utilization efficiency of wind energy, the wind turbines operate at the mode of maximum wind energy capture. As a result, the wind power fluctuates irregularly and randomly with the wind speed variation.

The volatility of wind speed has direct influence on the output power of wind turbines. The research on the characteristics of wind speed has been widely carried out to lay theoretical foundation for the real-time wind power suppression. Some researchers have proposed that the wind speed obeys the Gaussian, Chi-Square, Weibull or Rayleigh distribution [1-3]. The linear regression method, maximum likelihood method and Bayesian method were utilized to obtain the distribution function of wind speed. Furthermore, the clustering method was adopted to discuss the fluctuation of wind speed [4]. However, those above modelling methods emphasized the statistical description of the average wind speed within 10 minutes or longer.

With regard to the dynamic model of real-time wind speed, the model composed of basic component, gusty component, ramp component and random component was commonly adopted, especially in the simulation system [5]. However, the parameters in that model were hard to determine. The analysis on the operation data of wind farm was considered as another feasible approach to reveal the fluctuation characteristic of real-time wind power. In that field, the Poisson process and time sequence method were utilized by many researchers [6]. During the course of balancing fluctuant wind power in real-time, the calculation results obtained by that method often lag behind the variation of real-time wind power. As the main motion morphology of the atmosphere is turbulence, the turbulence model is considered as another effective way to describe the real-time wind speed [7]. With the occurrence of supersonic-flow anemometer, the real-time measurement and statistical analysis became the main method to study the property of atmospheric turbulence [8]. In USA and Norway, the database of local atmospheric turbulence has been established, which can precisely describe the fluctuation properties of real-time wind speed [9].

In this paper, we handle a research on the volatility of real-time wind speed based on the turbulence model. According to the turbulence model, the probability density function of fluctuant wind speed is deduced and the fluctuant wind power $\mathrm{P}_{\text {wave }}$ is decomposed into the steady fluctuation $\mathrm{P}_{\text {steady }}$ and peak fluctuation $\mathrm{P}_{\text {peak }}$. Also, the properties of $\mathrm{P}_{\text {steady }}$ and $\mathrm{P}_{\text {peak }}$ are investigated. Finally, we give a calculation example analysis on the decomposition of real-time fluctuant wind power.

\section{THE TURBULENCE MODEL OF REAL-TIME WIND SPEED}

In the wind farm, the wind driving the wind turbines belongs to the atmospheric boundary layer, where the material and energy exchange between the atmosphere and the earth surface take place. In the atmospheric boundary layer, the Reynolds number is large, and therefore the main motion morphology of the atmosphere is turbulence [7].

The turbulence is in nature complex, unordered and universal [10]. Meanwhile, the time and space scale of turbulence are broad. In 1973, Taylor and Kármá defined the turbulence as an unordered and random fluid motion in time and space domain [11]. The similarity theory of atmospheric boundary layer proposed by Monin and Obukhov laid theoretical foundation for the establishment of turbulence theory of the atmospheric boundary layer [12]. Some researches suggest that the atmospheric turbulence has the characteristics as follows [13]: 
i) In time and space domain, the atmospheric turbulence follows an unordered and random fluid motion.

ii) The whirlpool is the main movement pattern, accompanied by the inter-reaction between vorticity and strain.

iii) Its behavior is unrepeatable. However, its statistic property is predictable.

iv) The atmospheric turbulence is intermittent.

Reynolds proposed that the turbulence could be decomposed into the average and the fluctuation components [14]. Consequently, the real-time wind speed $V$ can be decomposed into the average wind speed $\bar{V}$ and the fluctuant wind speed $\Delta V$ [15]. $\bar{V}$ which varies slightly represents the average of the air velocity within ten minutes. In a certain time length, $\bar{V}$ can be considered as a constant. However, $\Delta V$ induced by the irregular motion in atmospheric turbulence often varies frequently and rapidly and can be considered to be nonlinear and fluctuant in time and space domain. As a result of the existence of $\Delta V$, the real-time wind speed $V$ in wind farm fluctuates irregularly and intermittently along the average value determined by $\bar{V}$. In general, the fluctuant wind speed $\Delta V$ regarded as the stable ergodic random process follows the Gauss distribution [15]. Therefore, the probability density function of $\Delta V$ is expressed by (1)

$$
f_{\Delta V}(x)=\frac{1}{\sqrt{2 \pi} \sigma} e^{-\frac{x^{2}}{2 \sigma^{2}}}
$$

Based on (1), we can carry out researches on the stochastic volatility of real-time wind speed.

\section{THE VOLATILITY OF REAL-TIME WIND POWER}

In order to fully exploit the wind energy, the variable speed pitch regulated wind turbine is widely constructed in the wind farm. The variable speed pitch regulated wind turbine often operates at the maximum wind energy capture mode. At that mode, the wind power varies with the wind speed.

According to the turbulence model of the real-time wind power, $\bar{V}$ represents the average speed of air flow in the wind farm. Thus, $\bar{V}$ determines the average power of wind farm. According to $\bar{V}$, we can establish the reference power value $P_{\text {ref }}$ for the wind farm. At the same time, the real-time wind speed in wind farm contains the massive fluctuation component $\Delta V$ which often accounts for $50 \%$ of the real-time wind speed. As a result, the wind power fluctuates irregularly and rapidly to a large extent along with the reference value $P_{\text {ref }}$ determined by $\bar{V}$.

\section{THE DECOMPOSITION OF WIND POWER FLUCTUATION BASED ON TURBULENCE MODEL}

As a kind of power source in power grid, the wind farm should be able to offer constant and controllable power supply to the power gird. Consequently, we do research on the decomposition of wind power fluctuation to lay a theoretical foundation for the real-time wind power regulation.

The real-time power generated by the wind turbine operating at the maximum wind energy capture mode contains the average and fluctuant component, as shown in (2).

$$
P_{\text {real }}=P_{\text {wave }}+P_{\text {ref }}
$$

Where $P_{\text {ref }}$ and $P_{\text {wave }}$ are caused by $\bar{V}$ and $\Delta V$ respectively. Generally, $\bar{V}$ can be obtained by the wind speed forecasting. Therefore, $P_{r e f}$ can be considered as the reference value for wind power generation. Under the maximum wind energy capture mode, $\Delta V$ makes the realtime wind power deviate from $P_{\text {ref }}$. The $P_{\text {wave }}$ induced by $\Delta V$ is just the fluctuant wind power needing to be regulated in real time. The value and probability distribution of $\Delta V$ will have a direct influence on $P_{\text {wave }}$.

As discussed above, $\Delta V$ follows the Gauss distribution. Then, we can derive the distribution function of the amplitude of $\Delta V(|\Delta V|)$. Assuming that the distribution function of $|\Delta V|$ is $F_{|\Delta V|}(Y), Y=|\Delta V| . F_{|\Delta V|}(Y)$ can be derived as follows:

$$
\begin{aligned}
F_{|\Delta V|}(Y) & =P(Y \leq y) \\
& =P(|\Delta V| \leq y) \\
& =P(-y \leq \Delta V \leq y) \\
& =P(\Delta V \leq y)-P(\Delta V \leq-y)
\end{aligned}
$$

According to (1), $P(\Delta V \leq y)$ and $P(\Delta V \leq-y)$ can be obtained separately.

$$
\begin{gathered}
P(\Delta V \leq y)=\int_{-\infty}^{y} f_{\Delta V}(x) \mathrm{d} x \\
=\int_{-\infty}^{y} \frac{1}{\sqrt{2 \pi} \sigma} e^{-\frac{x^{2}}{2 \sigma^{2}}} \mathrm{~d} x \\
P(\Delta V \leq-y)=\int_{-\infty}^{-y} f_{\Delta V}(x) \mathrm{d} x \\
=\int_{-\infty}^{-y} \frac{1}{\sqrt{2 \pi} \sigma} e^{-\frac{x^{2}}{2 \sigma^{2}}}
\end{gathered}
$$


Substituting (4) and (5) into (3) and performing derivation calculus, (6) is deduced.

$$
\begin{aligned}
F_{|\Delta V|}^{\prime}(Y) & =\frac{\mathrm{d} \int_{-\infty}^{y} \frac{1}{\sqrt{2 \pi} \sigma} e^{-\frac{x^{2}}{2 \sigma^{2}}} \mathrm{~d} x}{\mathrm{~d} y}-\frac{\mathrm{d} \int_{-\infty}^{-y} \frac{1}{\sqrt{2 \pi} \sigma} e^{-\frac{x^{2}}{2 \sigma^{2}}} \mathrm{~d} x}{\mathrm{~d} y} \\
& =\frac{\sqrt{2}}{\sqrt{\pi} \sigma} e^{-\frac{y^{2}}{2 \sigma^{2}}}
\end{aligned}
$$

As $F_{|\Delta V|}^{\prime}(Y)=f_{|\Delta V|}(y)$, the probability density function of $|\Delta V|$ can be given as

$$
f_{|\Delta V|}(y)=\frac{\sqrt{2}}{\sqrt{\pi} \sigma} e^{-\frac{y^{2}}{2 \sigma^{2}}} \quad y \geq 0
$$

By (7), the probability density curve of $|\Delta V|$ can be obtained as shown in Fig. 1 where $\sigma_{3}>\sigma_{2}>\sigma_{1}$.

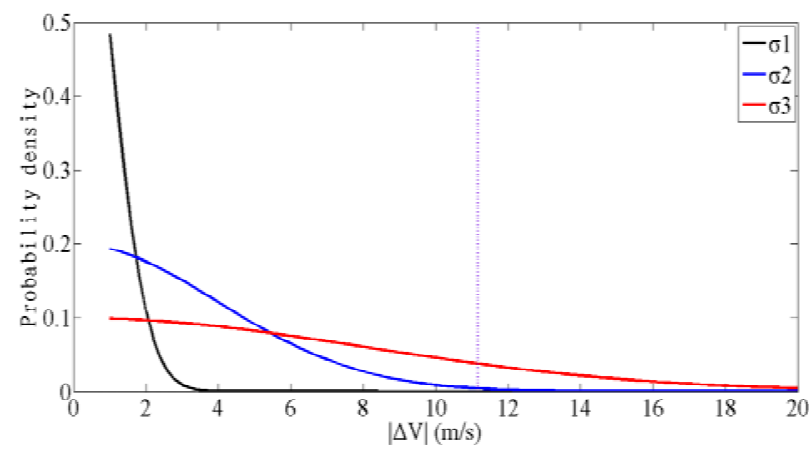

Figure 1. Probability density curve of $|\Delta \mathrm{V}|$

According to Figure 1, it can be seen that the probability decreases with the increase of $|\Delta V|$, tending towards zero gradually.

When the wind turbines operate at the mode of maximum wind energy capture, the real-time wind power is proportional to the cube of wind speed. Therefore, the wind power fluctuation will be slight when $|\Delta V|$ is small. On the contrary, the wind power will fluctuate heavily when $|\Delta V|$ is large. According to the distribution characteristic of $|\Delta V|$ mentioned above, the wind power fluctuation can be decomposed as follows:

i) The steady fluctuation $P_{\text {steady }} \cdot P_{\text {steady }}$ is caused by the $\Delta V$ whose amplitude is relatively small. Consequently, the amplitude of $P_{\text {steady }}$ is small. However, the occurrence probability of $P_{\text {steady }}$ is great because smaller $|\Delta V|$ is more likely to happen. With the frequent emergence of $P_{\text {steady }}$, $P_{\text {steady }}$ will cause great energy variation.

ii) The peak fluctuation $P_{\text {peak }} . P_{\text {peak }}$ is induced by the $\Delta V$ whose amplitude is large. According to Figure 1, the $\Delta V$ with the large amplitude seldom occurs. As a result, the $P_{\text {peak }}$ has large amplitude and small occurrence probability (towards zero). Correspondingly, the energy variation induced by $P_{\text {peak }}$ is small.

The battery-supercapacitor hybrid energy storage system is considered as an effective pattern to suppress the fluctuant wind power in real-time. Based on the wind power decomposition, in the battery-supercapacitor hybrid system, the battery can be allocated properly to balance the steady fluctuation $P_{\text {steady }}$. Therefore, the battery can fully develop its advantage of high energy density. As the supercapacitor has the properties of high power density and long cycle life, the supercapacitor in the hybrid system is arranged to regulate the peak fluctuation $P_{\text {peak }}$. By that task assignment for wind power suppression, the energy storage merits of battery and supercapacitor can be fully utilized. Consequently, the investment and maintenance cost for energy storage system can be reduced.

\section{THE EXAMPLE ANALYSIS ON THE WIND POWER DECOMPOSITION}

An example analysis is carried out to illuminate the wind power decomposition. In this example, the employed statistical method is the Byrne method.

In Figure 2, the operation power data of a wind farm within 24 hours is displayed.
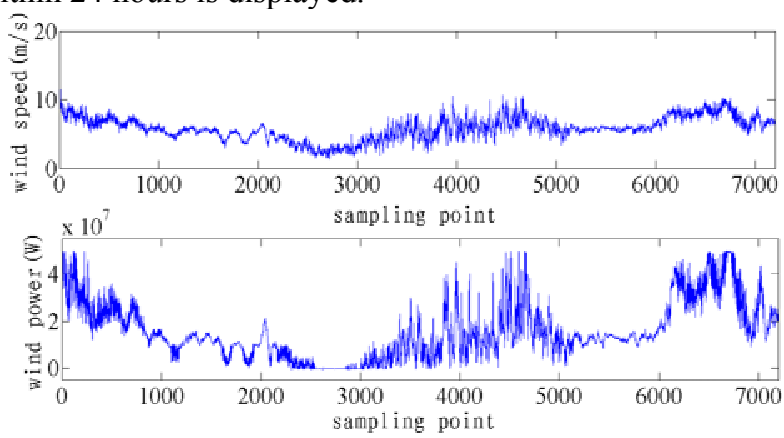

Figure 2. The curves of daily wind speed and output power of certain wind farm

The sampling interval is 12 seconds. Therefore, the data size in Fig. 3 is 7200 . In this example, we set the real-time reference value $P_{\text {ref }}$ of wind power to be the mean value of the data every 15 minutes. According to (2), the fluctuant wind power $P_{\text {wave }}$ can be obtained. 
Considering the aggregation of the absolute value of $P_{\text {wave }}$ as a sample $\mathrm{X} . \mathrm{X}$ is processed by the Byrne method as follows. Firstly, the maximum data $x_{\max }$ and minimum data $X_{\min }$ in $\mathrm{X}$ are obtained. Secondly, the data space $\left[\begin{array}{ll}X_{\min } & X_{\max }\end{array}\right]$ is divided into $M$ sections. Thirdly, the number $N$ of the data locating in each section is calculated. Finally, the frequency histogram of $\left|P_{\text {wave }}\right|$ is obtained by $N / n$. According to the grouping method proposed by the mathematician Sturges, $M$ is determined by (8).

$$
M=1+3.322 \lg (n)
$$

According to (8), $M=12$. By the Byrne method, the frequency histogram of $\left|P_{\text {wave }}\right|$ are given in Figure 3.

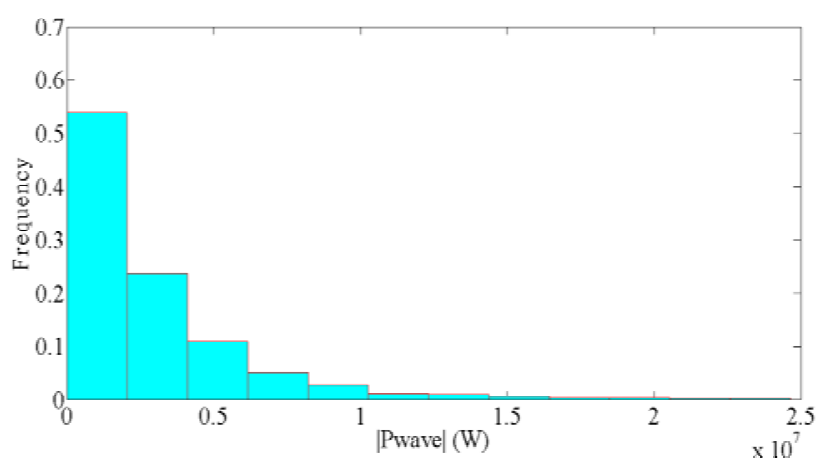

Figure 3. Frequency histogram of $\left|\mathrm{P}_{\text {wave }}\right|$.

As shown in Figure 3, the majority of the data in $\left|P_{\text {wave }}\right|$ is within the range of $0.002 \sim 12.324 \mathrm{MW}$. The amount of the data within that scope accounts for $97.5 \%$ of the data size of $\mathrm{X}$. Other data with the larger value accounts for $2.5 \%$ of the data size of $\mathrm{X}$. According to the properties of $P_{\text {steady }}$ and $P_{\text {peak }}$, the data within the range of $0.002 \sim 12.324 \mathrm{MW}$ belongs to the steady fluctuation $P_{\text {steady }}$. At the same time, the data within the range of $12.324-24.647 \mathrm{MW}$ belongs to the peak fluctuation $P_{\text {peak }}$.

When the batter-supercapacitor hybrid energy storage system is utilized to regulate the fluctuant wind power in real-time, the battery and supercapacitor is assigned to balance $P_{\text {steady }}$ and $P_{\text {peak }}$ separately according to their own properties. Consequently, the capacity of battery and supercapacitor can be calculated according to the above power decomposition

\section{CONCLUSIONS}

Based on the turbulence model of wind speed, this paper decomposes fluctuant wind power into the steady fluctuation $P_{\text {steady }}$ and peak fluctuation $P_{\text {peak }}$ to reveal the characteristics of the real-time fluctuant wind power. The steady fluctuation $P_{\text {steady }}$ has the low power value and leads to great energy variation. However, the peak fluctuation $P_{\text {peak }}$ whose power value is high induces the small energy fluctuation. At the same time, a calculation example for power decomposition is given out. The decomposition on the wind power fluctuation could lay theoretical foundation for the real-time wind power suppression.

\section{ACKNOWLEDGEMENTS}

This work is supported by the scientific researching fund of State Grid Shandong Electric Power Research Institute

\section{CORRESPONDING AUTHOR}

Name: Peng $\mathrm{Yu}$

Email: benben2229@hotmail.com

Mobile phone: +8618663755629

\section{REFERENCES}

[1] Jangamshetti S H and Ran V G, Optimum siting of wind turbine generators, IEEE Transactions on Energy Conversion, 16(1), pp.8-13, 2001.

[2] Youhua Hou, Dazhong Fang, Jun Qi, Hongbo Li, Wei Niu and Tao Yang, Analysis on active power fluctuation characteristics of largescale grid-connected wind farm and generation scheduling simulation under different capacity power injected from wind farms into power grid, Power System Technology, 34(5), pp.60-66, 2010.

[3] Chiodo E amd Lauria D, Bayes prediction of wind gusts for Wind Power Plants Reliability Estimation, 2011 International Conference on Clean Electrical Power, Ischia, Italy, 14 Jun - 16 Jun 2011, pp. 498-506.

[4] Jinbao Liu and Tao Ding, Wind speed prediction model based on radial basis functional neural network, Acta Energiae Solaris Sinica, 32(4), pp.560-564, 2011.

[5] Nichita C, Luca D, Dakyo B and Ceanga E, Large band simulation of the wind speed for real time wind turbine simulators, IEEE Transactions on Energy Conversion, 17(4), pp.523-529, 2002.

[6] Tao Han, Jiping Lu and Liang Qiao, Optimized scheme of energystorage capacity for grid-connected large-scale wind farm, Power System Technology, 34(1), pp.169-173, 2010.

[7] Leithead W E, de la Salle S and Reardon D, Role and objectives of control for wind turbines, IEE ProceedingsGeneration,Transmission\&Distribution, 138(2), pp.135-148, 1991.

[8] Roland B Stull, An introduction to boundary layer meteorology, pp.196, 1991.

[9] Liren $\mathrm{Xu}$, Zongkai Xu and Hongsheng Zhang, The research on the multiscale properties of the surface layer turbulence located on different underlying surface, Acta Meteorologica Sinica, 58(1), pp.84-93, 2000.

[10] Friedrich $\mathrm{H}$ Busse, Visualizing the dynamics of the onset of turbulence, Science, 305(5690), pp.1574-1575, 2004.

[11] Alexander Groisman and Victor Steinberg, Elastic turbulence in a polymer solution flow, Nature, 2000(405), pp.53-62, 2000. 
[12] Monin A S and Obukhov A M, Basic laws of turbulent mixing in the atmosphere near the ground, Tr Akad Nauk SSSR Geofiz Inst, 24(151), pp. 87-163, 1954.

[13] Businger J A, Wangaard J C and Izumi Y, Flux profile relationships in the atmospheric surface layer, Journal of Atmospheric Sciences, 28(2), pp.181-189,1971.
[14] Song Fu, Second-order moment closure model of turbulence probability density function, ACTA AERODYNAMICA SINICA, 13(1), pp.19-26,1995.

[15] E Welfonder, $\mathrm{R}$ Neifer and $\mathrm{M}$ Spanner, Development and experimental identification of dynamic models for wind turbines, Control Engineering Practice, 5 (1), pp.63-73,1997. 